\section{- OPEN ACCESS}

\title{
Cardiovascular disease risk scores in identifying future frailty: the Whitehall II prospective cohort study
}

\author{
Kim Bouillon, ${ }^{1}$ G David Batty, ${ }^{1,2}$ Mark Hamer, ${ }^{1}$ Severine Sabia, ${ }^{1}$ Martin J Shipley, ${ }^{1}$ \\ Annie Britton, ${ }^{1}$ Archana Singh-Manoux, ${ }^{1,3,4}$ Mika Kivimäki ${ }^{1,5}$
}

\begin{abstract}
- Additional material is published online only. To view please visit the journal online (http://dx.doi.org/10.1136/ heartjnl-2012-302922).

1 Department of Epidemiology and Public Health, University College London, London, UK ${ }^{2}$ Centre for Cognitive Ageing and Cognitive Epidemiology, University of Edinburgh, Edinburgh, UK

${ }^{3}$ INSERM U1018, Centre for Research in Epidemiology and Population Health, Hôpital Paul Brousse, Villejuif, France ${ }^{4}$ Centre de Gérontologie, Hôpital Ste Périne, AP-HP, Paris, France

${ }^{5}$ Finnish Institute of Occupational Health, Helsinki, Finland
\end{abstract}

\section{Correspondence to} Dr Kim Bouillon, Department of Epidemiology and Public Health, University College London, 1-19 Torrington Place, London WC1E 6BT, UK; kim.bouillon.09@ucl.ac.uk

Received 17 August 2012 Revised 27 December 2012 Accepted 28 December 2012 Published Online First 16 March 2013

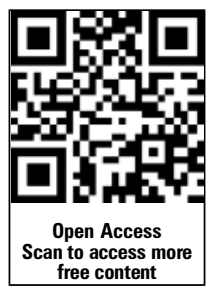

To cite: Bouillon $\mathrm{K}$, Batty GD, Hamer $M$, et al. Heart 2013;99:737-742.
ABSTRACT

Objectives To examine the capacity of existing cardiovascular disease (CVD) risk algorithms widely used in primary care, to predict frailty.

Design Prospective cohort study. Risk algorithms at baseline (1997-1999) were the Framingham CVD, coronary heart disease and stroke risk scores, and the Systematic Coronary Risk Evaluation.

Setting Civil Service departments in London, UK. Participants 3895 participants (73\% men) aged 45-69 years and free of CVD at baseline.

Main outcome measure Status of frailty at the end of follow-up (2007-2009), based on the following indicators: self-reported exhaustion, low physical activity, slow walking speed, low grip strength and weight loss. Results At the end of the follow-up, 2.8\% $(n=108)$ of the sample was classified as frail. All four CVD risk scores were associated with future risk of developing frailty, with ORs per one SD increment in the score ranging from $1.35(95 \% \mathrm{Cl} 1.21$ to 1.51$)$ for the Framingham stroke score to 1.42 (1.23 to 1.62) for the Framingham CVD score. These associations remained after excluding incident CVD cases. For comparison, the corresponding ORs for the risk scores and incident cardiovascular events varied between 1.36 (1.15 to 1.61) and 1.64 (1.50 to 1.80) depending on the risk algorithm.

Conclusions The use of CVD risk scores in clinical practice may also have utility for frailty prediction.

\section{INTRODUCTION}

Frailty is a clinically recognised geriatric syndrome characterised by declines in functioning across an array of physiological systems. ${ }^{1}$ Common symptoms of frailty are weight loss, exhaustion, low physical activity, slow walking speed at 'usual pace' and low grip strength. ${ }^{1}$ In the elderly, there is growing evidence that frailty predicts various adverse health outcomes such as disability, ${ }^{2}$ institutionalisation, ${ }^{2}$ falls, ${ }^{3}$ fractures, ${ }^{3}$ hospitalisation ${ }^{4}$ and mortality. ${ }^{3}$ In order to design interventions for preventing frailty, it is important to identify individuals at risk of developing the syndrome.

In addition to cardiovascular disease (CVD), there is increasing evidence to suggest that CVD risk factors measured in midlife predict a wide range of old-age health outcomes including cognitive decline and dementia, ${ }^{5}$ late-life depression ${ }^{6}$ and disability. ${ }^{7}$ Although few large-scale prospective studies have examined the association between
CVD risk factors and frailty, such a link is plausible for at least two reasons. First, several studies have shown a cross-sectional association between CVD and frailty. ${ }^{2}$ In one cross-sectional study, subclinical CVD diagnosed using non-invasive testing (carotid ultrasound, ankle-arm index, electrocardiography, echocardiography and cerebral MRI) was related to frailty after excluding clinically diagnosed CVD. ${ }^{8}$ Second, several individual risk factors included in multi-factorial prediction algorithms of CVD, such as the Framingham score, have been associated with frailty status: high blood pressure, ${ }^{9}$ diabetes, ${ }^{9}$ low high-density lipoprotein (HDL)-cholesterol level ${ }^{10}$ and cigarette smoking. ${ }^{11}$

In this study, we hypothesised that CVD risk scores used to assess 10-year risk of CVD would be associated with subsequent frailty status in people who were initially CVD-free. If a strong association between CVD risk scores and frailty is confirmed, these scores, importantly already routinely administered in clinical practice, would present a convenient way to identify individuals at an increased risk of frailty later in life and in need of early preventive measures. Evidence from randomised controlled trials suggest that exercise programmes ${ }^{12}$ and selected drugs (eg, dehydroepiandrosterone ${ }^{13}$ and testosterone ${ }^{14}$ ) can reverse frailty.

\section{METHODS}

Study population

Data were drawn from the Whitehall II study, an ongoing longitudinal study of 10308 (67\% men) London-based British civil servants aged 3555 years in $1985 .{ }^{15}$ Study inception (phase 1) took place during 1985-1988 and involved a clinical examination and self-administered questionnaire. Subsequent phases of data collection have alternated between postal questionnaire alone (phases 2 (1988-1990), 4 (1995-1996), 6 (2001), 8 (2006) and 10 (2011)), and postal questionnaire accompanied by a clinical examination approximately every 5 years (phases 3 (1991-1993), 5 (19971999), 7 (2002-2004) and 9 (2007-2009)).

We utilised CVD risk factors measured at phase 5 ('baseline' for the purposes of our analyses) to assess the risk of developing frailty at phase 9 when the frailty components were first measured. This design provides a 10 -year follow-up corresponding to that of the CVD risk prediction models we utilised. ${ }^{16-19}$ 


\section{CVD risk factors at baseline}

Blood samples were collected following either an 8-h overnight fast or at least a 4-h fast after a light fat-free breakfast. Serum for lipid analyses was refrigerated at $-4^{\circ} \mathrm{C}$ and assayed within $72 \mathrm{~h}$. Total cholesterol was determined by an enzymatic procedure using the automated cholesterol oxidase-phenol aminophenazone (CHOD-PAP) method. Serum HDL-cholesterol concentrations were measured from the supernatant after precipitation of non-HDL-cholesterol with phosphotungstate. Systolic blood pressure was measured twice with the Hawksley random zero sphygmomanometer in the sitting position after $5 \mathrm{~min}$ rest. We used the average of the two readings in the present analyses. Participants reported the medications used in the previous 14 days; responses were coded using the British National Formulary codes. ${ }^{20}$ Antihypertensive therapy was based on the use of the following drugs: diuretics, $\beta$-blockers, ACE inhibitors, calcium channel blockers and other antihypertensive drugs. Current smoking (yes/no) was ascertained by selfreport. Prevalent diabetes mellitus was defined based on reported doctor-diagnosed diabetes mellitus or use of diabetes medication, or when participants had a baseline fasting plasma glucose level $>126 \mathrm{mg} / \mathrm{dl}(>7.0 \mathrm{mmol} / \mathrm{l}){ }^{21}$ Presence of atrial fibrillation and left ventricular hypertrophy was determined on the ECG using the Minnesota Code: ${ }^{22}$ atrial fibrillation is coded as 8-3-1 and left ventricular hypertrophy as 3-1-0.

\section{CVD risk scores at baseline}

In addition to first relating individual CVD risk factors to later frailty risk, we also examined the predictive capacity of four established CVD risk score algorithms: the Framingham CVD ${ }^{18}$ coronary heart disease (CHD), ${ }^{19}$ stroke prediction models ${ }^{17}$ and SCORE (systematic coronary risk evaluation). ${ }^{16}$ Table 1 summarises all components included in the models, described below.

\section{Outcomes at follow-up}

Frailty was measured using the Fried frailty scale at the end of follow-up (phase 9, 2007-2009). This measure comprises the following components: self-reported exhaustion, low physical activity, slow walking speed, low grip strength and weight loss (cut-offs for each component are based on that of Fried et al). ${ }^{1}$ A total frailty score was calculated by allocating a value of 1 to each of the criteria if present, resulting in a range of $0-5$. Participants were classified as 'frail' if they had at least three out of five of the frailty components; as 'pre-frail' if they had 1-2; and as 'non-frail' if they had none of these components. ${ }^{1}$ Validated CVD outcomes (non-fatal CHD, non-fatal stroke, and a composite of non-fatal CVD cases including both groups) were assessed over the follow-up period (1997-1999 to 20072009). More details are available in the supplementary web appendix.

\section{Statistical analyses}

Each CVD risk factor at baseline was described according to the frailty status (frail, pre-frail, and non-frail) at year 10 of follow-up using the $\chi^{2}$ test, Fisher's exact test or analysis of variance as appropriate. We then summarised these associations using binary logistic regression analyses with frailty status dichotomised: frail versus pre-frail/non-frail. As the mean risk scores in men were systematically higher than those in women ( $\mathrm{p}$ values for all four scores $<0.0001$ ), we standardised these risk scores into standard scores (mean $=0, \mathrm{SD}=1$ ) in men and women separately. The OR of being frail or pre-frail was estimated per one SD increase (higher score represents greater CVD risk) in the risk scores over the 10-year follow-up. As sex did not modify the relation of the standardised risk scores with frailty at follow-up (all $\mathrm{p}$ values for sex interaction $>0.61$ ), men and women were combined in the analysis.

In examining the associations between individual risk factors and later frailty, we initially produced sex-adjusted models and then adjusted for the other risk factors to explore the independent effect of individual CVD risk factors with frailty. Binary logistic regression models were then used to examine the impact of a one SD increment in the risk scores on frailty at follow-up. We also examined the association between the CVD risk scores and incident cardiovascular events (CVD, CHD and strokes) to compare the strength of their associations to that with frailty. In addition, we conducted several sensitivity analyses. (1) To examine whether the association between the risk scores and frailty was mediated by underlying CVD, we estimated the strength of this association after excluding incident CVD cases. (2) To examine whether the association between the risk scores and frailty was biased by missing data, we imputed data for missing frailty status and individual CVD risk factors included in the risk scores. This was done for participants eligible at phase 5 and alive at the end of follow-up $(n=7412)$ using the method of multiple imputation by chained equations performed with an SAS-callable software application, IVEware. ${ }^{23}$ (3) We tested whether the CVD risk scores also predict 'pre-frailty' in a cohort excluding the frailty cases (see supplementary web appendix). Finally, to explore the extent to which the relationship between the risk scores and frailty was driven by specific CVD risk factors included in the scores, analyses on the risk scores-frailty associations were adjusted individually for each of their risk factors (see supplementary web appendix, table S1). A greater attenuation in the association after adjustment indicates a greater contribution of that specific risk factor. All analyses were performed with SAS V.9.1.

\section{RESULTS}

Of the 7870 study members who participated at phase 5 , a total of 3895 participants (1037 women) aged 45-69 years constituted the analytic sample (figure 1). Compared with participants

Table 1 Composition of the SCORE and Framingham CVD, CHD and stroke risk algorithms

\begin{tabular}{|c|c|c|c|c|c|c|c|c|c|c|c|c|c|}
\hline Score & Country & Sex & Age & Total C & HDL-C & SBP & DBP & AHTD & Smoking & Diabetes & CVD & $\mathrm{AF}$ & LVH \\
\hline Framingham CVD & USA & + & + & + & + & + & & + & + & + & & & \\
\hline Framingham CHD & USA & + & + & + & + & + & + & & + & + & & & \\
\hline Framingham stroke & USA & + & + & & & + & & + & + & + & + & + & + \\
\hline SCORE & Europe & + & + & + & & + & & & + & & & & \\
\hline
\end{tabular}

AHTD, antihypertensive drug; AF, atrial fibrillation; CVD, cardiovascular disease; CHD, coronary heart disease; C, cholesterol; DBP, diastolic blood pressure; LVH, left ventricular hypertrophy; SBP, systolic blood pressure; SCORE, Systematic Coronary Risk Evaluation. 


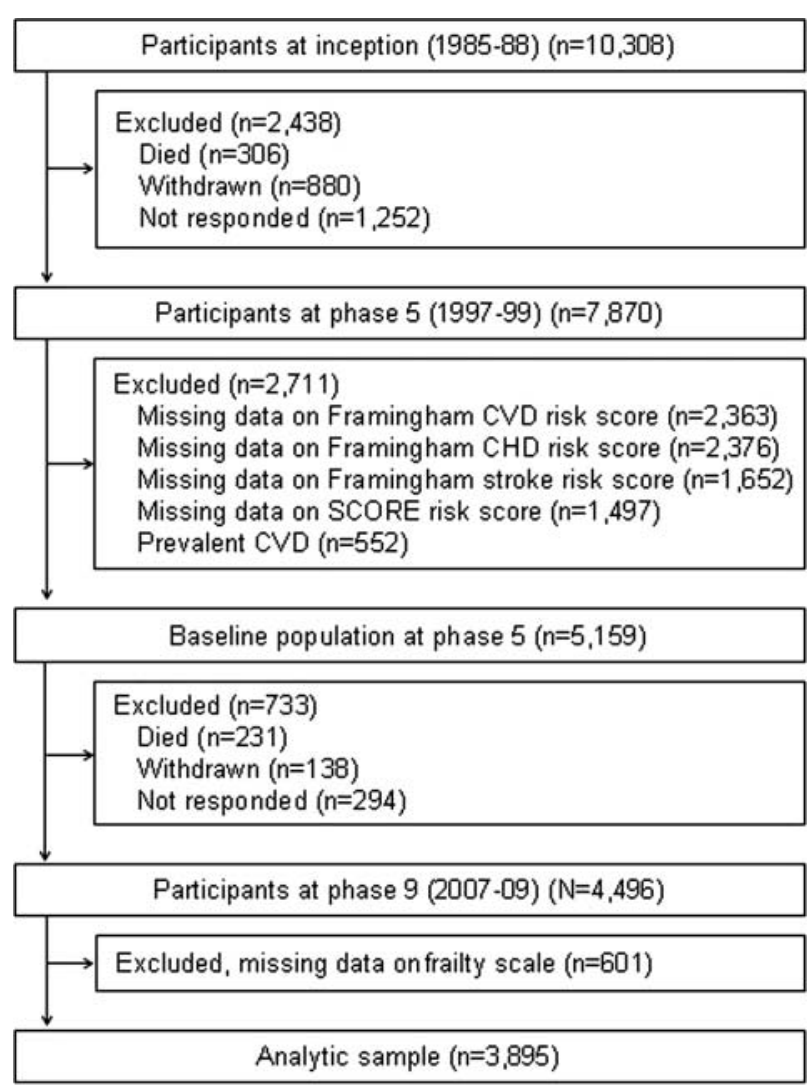

CVD: cardiovascular disease; CHD: coronary heart disease; SCORE: Systematic Coronary Risk Evaluation

Figure 1 Flow of study members through the data collection phases in Whitehall II.

alive at phase 9 but excluded (owing to non-participation at phases 5 and 9, prior history of CVD at phase 5, and missing data on the CVD risk scores or the frailty scale, total $n=3517$ ), persons included in the analytic sample $(n=3895)$ were 1.1 years younger $(p<0.0001)$, less likely to be female $(26.6 \%$ vs $34.5 \%, \mathrm{p}<0.0001)$ and less likely to be from the lower socioeconomic group $(10.0 \%$ vs $20.0 \%, \mathrm{p}<0.0001)$ (see supplementary web appendix, table S2).

In table 2 we present the baseline characteristics of participants according to frailty status at the end of follow-up, on average 10.5 years $(\mathrm{SD}=0.5)$ after the measurement of CVD risk scores. Of the 3895 participants, $2.8 \%$ were classified as frail, $37.1 \%$ as pre-frail and $60.1 \%$ as non-frail. In comparison with non-frail participants, frail participants were more likely to be older, female, use antihypertensive treatment, smoke and have diabetes. Frail participants were also more likely to have experienced a CVD event during the follow-up relative to their non-frail counterparts (incidence $16.7 \%$ vs $8.5 \%, \mathrm{p}=0.01$ ).

In table 3 , we present the association between the individual CVD risk factors at baseline and frailty at follow-up. In the model including all risk factors, only two were independently associated with future frailty: increased age and use of antihypertensive treatment. Thus, a one SD increment in age (5.9 years for men and women) increased the odds of being frail by $56 \%(\mathrm{OR}=1.56,95 \% \mathrm{CI} 1.28$ to 1.92$)$ and using an antihypertensive treatment increased the odds by $77 \%(\mathrm{OR}=1.77$, 95\% CI 1.10 to 2.94$)$.
Table 4 shows the association of a one SD increment in the CVD risk scores with future frailty and cardiovascular events. All risk scores had a similar strength of association with frailty, with the ORs ranging from 1.35 (95\% CI 1.21 to 1.51 ) for the Framingham stroke risk score to 1.42 (95\% CI 1.23 to 1.62 ) for the Framingham CVD risk score. As expected, the association of the CVD risk scores was stronger in relation to predicting CVD events, with ORs ranging from 1.36 (95\% CI 1.15 to 1.61$)$ for the Framingham stroke risk score to 1.64 (95\% CI 1.50 to 1.80) for the Framingham CVD risk score. The strength of the association between the CVD risk scores and frailty remained essentially the same after exclusion of incident CVD cases, and in multiple imputation (see supplementary web appendix, table S3). The CVD risk scores also predicted pre-frailty although to a lesser extent than for frailty (see supplementary web appendix, table S4).

In supplementary web appendix, table S1, we present results of analyses in which the four CVD risk scores were adjusted for each of their risk factors. The association between risk scores and frailty was attenuated after adjustments for age and antihypertensive treatment, but is still statistically significant, suggesting that this association was not driven by any specific risk factor.

\section{DISCUSSION}

Our main finding from this cohort of middle aged individuals was that four different CVD risk scores were associated with an elevated risk of frailty. Thus, one sex-specific SD increment in the risk scores increased the odds of being classified as frail at the end of the 10 -year follow-up by $35-42 \%$. The strength of this association was only slightly diminished after exclusion of cases of CVD during the follow-up, suggesting that the predictive risk score-frailty associations were not driven by co-morbid CVD. Furthermore, we found that these scores stratified the risk of developing frailty. To the best of our knowledge, the link between scores from CVD risk factor engines and future frailty has not been examined.

Although initially designed to predict CVD, our results suggest that the CVD risk scores also appear to be a predictive marker of general health such as frailty status. In a previous study, the Framingham CVD risk score was also found to be associated with cognitive decline. ${ }^{5}$ Our finding in relation to frailty is plausible given that each risk factor-age, total cholesterol, HDL-cholesterol, systolic blood pressure, smoking and diabetes-included in these scores has also been shown to be associated with various other health outcomes including cancer, which, after CVD, is the second leading cause of death in economically developed countries. ${ }^{24}$ One plausible mechanism linking risk scores to both CVD and frailty is the presence of atherosclerosis in arteries and related chronic systemic inflammation. $^{25}$ Atherosclerotic processes can prevent blood flow through the coronary artery, causing CVD, ${ }^{25}$ and through the muscles, causing sarcopenia, a clinical feature of frailty. ${ }^{26}$

We found that the proportion of frailty was higher in women than men $(5.1 \%$ versus $1.9 \%$, respectively). This is in agreement with previous findings, ${ }^{27}$ but opposite to what one might expect for CVD, which is more common in men in late middle-age. In our study, the incidence of CVD was 9.9\% in men versus $5.7 \%$ in women. A potential explanation for the higher incidence of frailty in women pertains to differences in biology between the sexes, with men having greater bone mineral density and muscle mass in old age. ${ }^{28}$ 
Table 2 Characteristics of participants in the analytical sample $(n=3895)$

\begin{tabular}{|c|c|c|c|c|c|}
\hline & \multirow[b]{2}{*}{ All } & \multicolumn{3}{|c|}{ Frailty status at follow-up } & \multirow[b]{2}{*}{ p Value* } \\
\hline & & Not frail & Pre-frail & Frail & \\
\hline Number & 3895 & 2342 & 1445 & 108 & \\
\hline Age, years, mean (SD) & $55.2(5.9)$ & $54.9(5.7)$ & $55.5(6.1)$ & $57.9(6.5)$ & $<0.0001$ \\
\hline \multicolumn{6}{|l|}{ Sex, $n(\%)$} \\
\hline Male & $2858(73.4)$ & $1821(77.8)$ & $982(68.0)$ & $55(50.9)$ & $<0.0001$ \\
\hline Female & $1037(26.6)$ & $521(22.4)$ & $463(32.0)$ & $53(49.1)$ & \\
\hline Total cholesterol, mmol/l, mean (SD) & $5.92(1.05)$ & $5.91(1.02)$ & $5.94(1.09)$ & $5.99(1.03)$ & 0.22 \\
\hline HDL cholesterol, mmol/l, mean (SD) & $1.46(0.39)$ & $1.47(0.39)$ & $1.45(0.38)$ & $1.47(0.39)$ & 0.21 \\
\hline Systolic blood pressure, mm Hg, mean (SD) & $122.7(16.0)$ & $122.3(15.7)$ & $123.3(16.3)$ & $124.5(16.1)$ & 0.03 \\
\hline Diastolic blood pressure, mm Hg, mean (SD) & $77.6(10.3)$ & $77.5(10.2)$ & $77.8(10.5)$ & $78.4(11.6)$ & 0.28 \\
\hline \multicolumn{6}{|l|}{ Antihypertensive treatment, $\mathrm{n}(\%)$} \\
\hline No & $3515(90.2)$ & $2137(91.3)$ & $1293(89.5)$ & $85(78.7)$ & $<0.0001$ \\
\hline Yes & $380(9.8)$ & $205(8.7)$ & $152(10.5)$ & $23(21.3)$ & \\
\hline \multicolumn{6}{|l|}{ Smoking, n (\%) } \\
\hline No & $3593(92.3)$ & 2185 (93.3) & $1313(90.9)$ & $95(88.0)$ & 0.006 \\
\hline Yes & $302(7.8)$ & $157(6.7)$ & $132(9.1)$ & $13(12.0)$ & \\
\hline \multicolumn{6}{|l|}{ Diabetes, n (\%) } \\
\hline No & 3755 (96.4) & $2273(97.1)$ & 1381 (95.6) & $101(93.5)$ & 0.02 \\
\hline Yes & $140(3.6)$ & $69(3.0)$ & $64(4.4)$ & $7(6.5)$ & \\
\hline \multicolumn{6}{|l|}{ Atrial fibrillation, $n(\%)$} \\
\hline No & $3882(99.7)$ & $2335(99.7)$ & 1439 (99.6) & $108(100.0)$ & - \\
\hline Yes & $13(0.3)$ & $7(0.3)$ & $6(0.4)$ & 0 & \\
\hline \multicolumn{6}{|l|}{ Left ventricular hypertrophy, $\mathrm{n}(\%)$} \\
\hline No & $3667(94.2)$ & $2214(94.5)$ & $1356(93.8)$ & $97(89.8)$ & 0.10 \\
\hline Yes & $228(5.8)$ & $128(5.5)$ & $89(6.2)$ & $11(10.2)$ & \\
\hline \multicolumn{6}{|l|}{ Incident CVD at follow-up, n (\%) } \\
\hline No & $3552(91.2)$ & $2143(91.5)$ & $1319(91.3)$ & $90(83.3)$ & 0.01 \\
\hline Yes & $343(8.8)$ & $199(8.5)$ & $126(8.7)$ & $18(16.7)$ & \\
\hline \multicolumn{6}{|l|}{ Incident CHD at follow-up, $\mathrm{n}(\%)$} \\
\hline No & $3582(92.0)$ & 2165 (92.4) & $1324(91.6)$ & $93(86.1)$ & 0.05 \\
\hline Yes & $313(8.0)$ & $177(7.6)$ & $121(8.4)$ & $15(13.9)$ & \\
\hline \multicolumn{6}{|l|}{ Incident stroke at follow-up, n (\%) } \\
\hline No & $3856(99.0)$ & $2316(98.9)$ & $1436(99.4)$ & $104(96.3)$ & 0.01 \\
\hline Yes & $39(1.1)$ & $26(1.1)$ & $9(0.6)$ & $4(3.7)$ & \\
\hline
\end{tabular}

This study has some limitations. First, we identified frailty cases by using a measure operationalised by Fried et al, ${ }^{1}$ but a recent review identified that there are more than 20 alternative measures of frailty. ${ }^{29}$ Although there are no gold standard measures, the measure by Fried et al is the most widely used. Second, we assessed CVD risk at the mean age of 55 years. It remains unclear whether our findings are generalisable to other age groups because at older ages low rather than high levels of some cardiovascular risk factors (total cholesterol, low-density lipoprotein (LDL)-cholesterol and systolic blood pressure) are associated with poor health outcome, as assessed by activity daily living disability, hospitalisation, functional performance and mortality. ${ }^{30}$ In relation to CVD prediction, the risk scores are not recommended to be used at older ages ( $>75$ years); the validity of these scores as risk markers of frailty should be examined in that age in future studies. Third, approximately half of the study members who participated at phase 5 were excluded from the analysis due to death, non-participation, loss to follow-up or missing data. Our sensitivity analysis suggests this is not a major source of bias because the results using the multiple multivariate imputation method were largely similar to those reported in the main analysis. However, we cannot rule out bias arising from attrition not covered by the missingness-at-random assumption. Finally, our study sample consisted of middle-aged civil servants, limiting the generalisability of our findings. These limitations can be compared to the main strength of our study, which resides in the use of prospectively collected data given that previous studies that have examined the association between CVD or its individual risk factors and frailty used cross-sectional data. ${ }^{2} 89$ Our results suggest a relationship between the CVD risk scores and frailty that is independent of existing CVD. However, these findings, based on observational data, do not provide information about causality as we cannot rule out the confounding effect of unmeasured risk factors.

Besides the clinical utility of CVD risk scores-Framingham CVD, CHD, stroke or SCORE-in predicting risk of cardiovascular death and disease, our results suggest that they may also help to identify middle-aged persons who will benefit from interventions designed to prevent frailty. As such, the use of CVD risk scores in clinical practice may also have utility for frailty prediction. 
Table 3 Association between individual cardiovascular disease risk factors at baseline and frailty at 10-year follow-up $(n=3895)$

\begin{tabular}{|c|c|c|c|}
\hline \multirow[b]{2}{*}{ Predictors } & \multirow[b]{2}{*}{ N (\%) } & \multicolumn{2}{|c|}{ OR $(95 \% \mathrm{Cl})$ for frailty } \\
\hline & & Adjusted for sex & Fully adjusted $t$ \\
\hline Age, years* & 3895 & 1.58 (1.30 to 1.91$)$ & 1.56 (1.28 to 1.92$)$ \\
\hline Total cholesterol, mg/d| ${ }^{*}$ & 3895 & $1.05(0.87$ to 1.26$)$ & $0.96(0.79$ to 1.18$)$ \\
\hline HDL cholesterol, mg/dl* & 3895 & 0.84 (0.69 to 1.03$)$ & $0.90(0.73$ to 1.10$)$ \\
\hline Systolic blood pressure, $\mathrm{mm} \mathrm{Hg}^{*}$ & 3895 & 1.15 (0.96 to 1.39$)$ & 0.87 (0.65 to 1.15$)$ \\
\hline Diastolic blood pressure, $\mathrm{mm} \mathrm{Hg}^{*}$ & 3895 & 1.17 (0.97 to 1.42$)$ & $1.20(0.91$ to 1.59$)$ \\
\hline \multicolumn{4}{|l|}{ Antihypertensive treatment } \\
\hline No & $3515(90.2)$ & 1 (ref) & 1 (ref) \\
\hline Yes & $380(9.8)$ & $2.42(1.50$ to 3.90$)$ & 1.77 (1.10 to 2.94$)$ \\
\hline \multicolumn{4}{|l|}{ Smoking } \\
\hline No & $3593(92.2)$ & 1 (ref) & 1 (ref) \\
\hline Yes & $302(7.8)$ & 1.50 (0.83 to 2.72$)$ & $1.62(0.88$ to 2.97$)$ \\
\hline \multicolumn{4}{|l|}{ Diabetes } \\
\hline No & 3755 (96.4) & 1 (ref) & 1 (ref) \\
\hline Yes & $140(3.6)$ & 1.81 (0.82 to 3.99$)$ & $1.29(0.57$ to 2.91$)$ \\
\hline \multicolumn{4}{|l|}{ Atrial fibrillation } \\
\hline No & 3882 (99.7) & - & - \\
\hline Yes & $13(0.3)$ & - & - \\
\hline \multicolumn{4}{|l|}{ Left ventricular hypertrophy } \\
\hline No & 3667 (94.1) & 1 (ref) & 1 (ref) \\
\hline Yes & $228(5.9)$ & 2.09 (1.10 to 3.97$)$ & $1.66(0.85$ to 3.21$)$ \\
\hline
\end{tabular}

Table 4 OR per one sex-specific SD increment in score using four CVD risk algorithms for future frailty and cardiovascular diseases ( $n=3895$ )

\begin{tabular}{|c|c|c|c|c|c|}
\hline & \multicolumn{2}{|l|}{ Frailty } & \multicolumn{3}{|c|}{ Cardiovascular disease } \\
\hline & Number of cases & OR $(95 \% \mathrm{Cl})$ & Outcome & Number of cases & OR $(95 \% \mathrm{Cl})$ \\
\hline Framingham CVD risk score & 108 & 1.42 (1.23 to 1.62$)$ & Any CVD & 343 & 1.64 (1.50 to 1.80$)$ \\
\hline Framingham CHD risk score & 108 & 1.38 (1.20 to 1.59$)$ & CHD & 313 & 1.53 (1.40 to 1.68$)$ \\
\hline Framingham stroke risk score & 108 & 1.35 (1.21 to 1.51$)$ & Stroke & 39 & 1.36 (1.15 to 1.61$)$ \\
\hline SCORE (CVD risk score) & 108 & $1.36(1.18$ to 1.56$)$ & Any CVD & 343 & 1.57 (1.44 to 1.71$)$ \\
\hline
\end{tabular}

CVD, cardiovascular disease; CHD, coronary heart disease; SCORE, Systematic Coronary Risk Evaluation.

Acknowledgements We thank all participating men and women in the Whitehall II study; all participating Civil Service departments and their welfare, personnel and establishment officers; the Occupational Health and Safety Agency; and the Council of Civil Service Unions. The Whitehall II study team comprises research scientists, statisticians, study coordinators, nurses, data managers, administrative assistants and data entry staff, who make the study possible.

Contributors MK and GDB conceived the idea for the study and along with KB developed the objectives and design of the study. $\mathrm{KB}$ ran the analyses and acts as guarantor of the paper. KB, MK and GDB drafted the paper. All authors contributed to the interpretation of results and revision of the paper, and approved the final version of the paper.

Funding This work was supported by grants from the Medical Research Council, UK; Economic and Social Research Council, UK; British Heart Foundation, UK; Health and Safety Executive, UK; Department of Health, UK; BUPA Foundation, UK; National Heart Lung and Blood Institute (R01HL036310), USA; NIH (National Institute on Aging) (R01AG013196; R01AG034454), USA. GDB is a Wellcome Trust Fellow, UK. MS is supported by the British Heart Foundation, ASM is supported by a 'European Young Investigator Award' from the European Science Foundation, and MK is supported by the UK Medical Research Council, the EU New OSH ERA research programme, the Academy of Finland, Finland and by a professorial fellowship from the Economic and Social Research Council, UK.

Competing interests None.

Ethics approval This study was approved by the University College London ethics committee, and participants provided written consent.

Provenance and peer review Not commissioned; externally peer reviewed.
Data sharing statement Whitehall II data, protocols, and other metadata are available to the scientific community. Please refer to the Whitehall II data sharing policy at http://www.ucl.ac.uk/whitehallll/data-sharing.

Open Access This is an Open Access article distributed in accordance with the Creative Commons Attribution Non Commercial (CC BY-NC 3.0) license, which permits others to distribute, remix, adapt, build upon this work non-commercially, and license their derivative works on different terms, provided the original work is properly cited and the use is non-commercial. See: http://creativecommons.org/ licenses/by-nc/3.0/

\section{REFERENCES}

1 Fried LP, Tangen CM, Walston J, et al. Frailty in older adults: evidence for a phenotype. J Gerontol A Biol Sci Med Sci 2001;56:M146-56.

2 Bandeen-Roche K, Xue QL, Ferrucci L, et al. Phenotype of frailty: characterization in the women's health and aging studies. J Gerontol A Biol Sci Med Sci 2006:61:262-6.

3 Ensrud KE, Ewing SK, Taylor BC, et al. Frailty and risk of falls, fracture, and mortality in older women: the study of osteoporotic fractures. J Gerontol A Biol Sci Med Sci 2007;62:744-51.

4 Rochat S, Cumming RG, Blyth F, et al. Frailty and use of health and community services by community-dwelling older men: the Concord Health and Ageing in Men Project. Age Ageing 2010;39:228-33.

5 Kaffashian $\mathrm{S}$, Dugravot $\mathrm{A}$, Nabi $\mathrm{H}$, et al. Predictive utility of the Framingham general cardiovascular disease risk profile for cognitive function: evidence from the Whitehall II study. Eur Heart J 2011;32:2326-32. 
6 Kivimaki M, Shipley MJ, Allan $\mathrm{CL}$, et al. Vascular risk status as a predictor of later-life depressive symptoms: a Cohort Study. Biol Psychiatry 2012;72:324-30.

7 Pinsky JL, Branch LG, Jette AM, et al. Framingham Disability Study: relationship of disability to cardiovascular risk factors among persons free of diagnosed cardiovascular disease. Am J Epidemiol 1985;122:644-56.

8 Newman AB, Gottdiener JS, McBurnie MA, et al. Associations of subclinical cardiovascular disease with frailty. J Gerontol A Biol Sci Med Sci 2001;56: M158-66.

9 Lee JS, Auyeung TW, Leung J, et al. Physical frailty in older adults is associated with metabolic and atherosclerotic risk factors and cognitive impairment independent of muscle mass. J Nutr Health Aging 2011;15:857-62.

10 Landi F, Russo A, Cesari M, et al. HDL-cholesterol and physical performance: results from the ageing and longevity study in the sirente geographic area (ilSIRENTE Study). Age Ageing 2007;36:514-20.

11 Strawbridge WJ, Shema SJ, Balfour JL, et al. Antecedents of frailty over three decades in an older cohort. J Gerontol B Psychol Sci Soc Sci 1998;53:S9-16.

12 Peterson MJ, Sloane R, Cohen HJ, et al. Effect of telephone exercise counseling on frailty in older veterans: project LIFE. Am J Mens Health 2007;1:326-34.

13 Kenny AM, Boxer RS, Kleppinger A, et al. Dehydroepiandrosterone combined with exercise improves muscle strength and physical function in frail older women. $J$ Am Geriatr Soc 2010;58:1707-14.

14 Kenny AM, Kleppinger A, Annis K, et al. Effects of transdermal testosterone on bone and muscle in older men with low bioavailable testosterone levels, low bone mass, and physical frailty. J Am Geriatr Soc 2010;58:1134-43.

15 Marmot M, Brunner E. Cohort Profile: the Whitehall II study. Int J Epidemiol 2005;34:251-6.

16 Conroy RM, Pyorala K, Fitzgerald AP, et al. Estimation of ten-year risk of fatal cardiovascular disease in Europe: the SCORE project. Eur Heart $J$ 2003:24:987-1003
17 D'Agostino RB, Wolf PA, Belanger AJ, et al. Stroke risk profile: adjustment for antihypertensive medication. The Framingham Study. Stroke 1994;25:40-3.

18 D'Agostino RB Sr, Vasan RS, Pencina MJ, et al. General cardiovascular risk profile for use in primary care: the Framingham Heart Study. Circulation 2008;117:743-53.

19 Wilson PW, D'Agostino RB, Levy D, et al. Prediction of coronary heart disease using risk factor categories. Circulation 1998;97:1837-47.

20 Joint Formulary Committee. British national formulary. 32 edn. London: BMJ Group and Pharmaceutical Press, 1996.

21 American Diabetes Association, Diagnosis and classification of diabetes mellitus Diabetes Care 2012:35:S64-71.

22 Prineas R, Crow R, Blackburn H. The Minnesota code manual of electrocardiographic findings. Littleton, Massachusetts, USA: John Wright-PSG, 1982.

23 Raghunathan TE, Solenberger PW, van Hoewyk J. IVEware: Imputation and Variance Estimation Software User Guide. http://www isr umich edu/src/smp/ive 2002 (accessed 1 Aug 2012).

24 World Health Organization. The global burden of disease 2004 update. Geneva: World Health Organization, 2008.

25 Hansson GK. Inflammation, atherosclerosis, and coronary artery disease. N Engl J Med 2005:352:1685-95

26 Morley JE, Perry HM III, Miller DK. Editorial: Something about frailty. J Gerontol A Biol Sci Med Sci 2002;57:M698-704.

27 Rockwood K. What would make a definition of frailty successful? Age Ageing 2005:34:432-4.

28 Newman AB, Brach JS. Gender gap in longevity and disability in older persons. Epidemiol Rev 2001;23:343-50.

29 Sternberg SA, Wershof Schwartz A, Karunananthan S, et al. The identification of frailty: a systematic literature review. J Am Geriatr Soc 2011;59:2129-38.

30 Schupf N, Costa R, Luchsinger J, et al. Relationship between plasma lipids and all-cause mortality in nondemented elderly. J Am Geriatr Soc 2005;53:219-26. 